Schweiz. Z. Path. Bakt. 1956;19:I-VIII

\title{
Contents, Vol. 19, 1956
}

Offizielles Organ der FreienVereinigung Schweizer Pathologen/ScL·weiz. Mikrobiologischen Gesellschi Organe officiel de ГAssociation libre des Pathologistes Suisses/la Société Suisse de Microbiologie

H. BERGSTRAND, Stockholm

R. BIELING, Wien

H. CHIARI, Wien

M. DJURISIC, Beograd

J. FIRKET, Liège

E. GRASSET, Geneve

C. HALLAUER, Bern

E. LETTERER, Tubingen

EDITORES

A. LINDAU, Lund

E. G. NAUCK, Hamburg

J. L. NICOD, Lausanne

CH. OBERLING, ParU

A. L. OLITZKI, Jerusalem

F. C. ROULET, Basel

E. RUTI6HAUSER, Geneve H. SMETANA, Washington

J. TOMCSIK, Basel

R. TULASNE, Strasbourg

E. UEHLINGER, Zurich

H. UN GAR, Jerusalem

B. WALTHARD, Bern

M. WELSCH, Liège

A. WERTHEMANN, Basel

K. C. WINKLER, Utrecht

REDACTORES A. v. ALBERTINI, Zurich A. GRUMBACH, Zurich H. MOOSER, Zurich

Vol. 19

1956

BASEL (Schweiz) S. KARGER NEW YORK

Index

Agnese, G.: RecheГches sur les porteurs de virus poliomyé-

litique 624

Albertini, A. v., M. N. Metaxas und M. Metaxas-Bühler: t)ber

das gleichzeitige Einsetzen von Allergie und Tuberkel-

bildung bei der Meerschweinchentuberkulose ... 1

Banic, S.: Isolation of the Streptomycin Resistant Variants 
of E. Coli B by a Modified Replica Plating Technique . 792

Barbíeri, A. de, et M. E. Scevola: Sur les modifications de

Tactivité de quelques systèmes enzymatiques de $\Gamma$ orga-

nisme animal provoquées par $\Gamma$ antigène $O$ et $\Gamma$ antigène

$\mathrm{Vi} \quad 576$

Barrier, F. R.: Über nekrotisierende Arteriitis nach Impfungen und ihre Deutung im Rahmen der heutigen Allergie-

lehre 411

Baumann-Grace, J. B.t, vide J. Tomcsík.

Bednáf, B., vide K. Raska.

Beer, K.: Über Torulose 534

Blondel, B., vide E. Grasset.

Brunner, H. E., vide J. R. Rüttner.

Buchner, P.: Die harmonische Einbürgerung pflanzlicher

Mikroorganismen in den tierischen Körper . . 664

Champendal,P.: Recherches bactériologiques à propos d'une

épidémie hospitalière de staphylococcies .... 497

Crovari, P., vide St. Kanítz.

Cruchaud, A.: Lesions articulaires et périarticulaires de

Thypovitaminose C chronique chez le Cobaye . . 369

Dekleva, A., and M. Likar: Isolation and Electron Microsco

pic Morphology of the Virus of Meningoencephalitis in

Slovenia 184

Desaulles, P., B. Schär und R. Meier: Wirkung von An-

thraxfraktionen auf mesenchymale Zellreaktionen in

vitro und in vivo 639

Dietrich, F. M.: Die Typisierung von pathogenen und apa-

thogenen Corynebakterien auf Grund der Kolonienmor-

phologie 461

Ettinger, L., und H. Zähner: Induzierte Pikromycin-Resi-

stenz bei Micrococcus pyogenes var. aureus . . 103

Alle Rechte, insbesondere das der Übersetzung in fremde Sprachen, vorbehalten.

Ohne ausdrückliche Genehmigung des Verlages ist es auch nicht gestattet, dieses Buch oder

Teile

daraus auf photomechanischem Wege (Photokopie, Mikrokopie) zu vervielfältigen.

(C)

Copyright 1956 by S. Karger AG., Basel Printed in Switzerland by Buchdruckerei Friedrich

Reinhardt AG., Basel. Cliches: Aberegg-Steiner \& Cie. AG., Bern, und Steiner \& Cie. AG.,

Basel

Kovács, K., and Z. B. Mónus: Diabetes Insipidus Syndrome

Developed with Myelocytic Leukaemia 278

Krech, U.: Adaptationsversuche in Mäusen mit Poliomye

litis-Virus Typ $1 \quad 628$

Kürsten, H., vide P. Speiser.

Kunz, W., vide L. Neipp.

Lavillaureix, J.: Les formes L des vibrions: Leur pouvoir 
pathogène 615

Likar, M., vide A. Dekleva. Lindenmann, J., vide H. Mooser. Lippeit, H., vide E. Mannweíler.

Mannweile $\Gamma$, E., und H. Líppelt: Untersuchungen zu $\gamma$ Sero-

diagnostik der Listeriose 758

Massini, M.-A.: Über die Sarkome der Schilddrüse mit be-

sonderer Berücksichtigung der Lympho- und Retothel-

sarkome 259

Meier, R., vide P. Desaulles.

- vide L. Neipp.

Metaxas, M. N., vide A. v. Alberiini.

Metzger, M.: New Mannitol Negative Variety of Shigella

Flexneri 2a 778

Meyer, K.|, vide G. A. Moosbrugger.

Meyer, K. F., vide M. Schär.

Meznik-Schönbauer, E., vide P. Speiser.

Milgrom, F., and Z. Swíerczyท́ska: Studies on the Specificity of Natural Antibacterial Antibodies .... 189

Molnár, J.: Über die dichroitische Färbung der Kapsel des

Cryptococcus neoformans 82

- $\quad$ Über die Struktur der Kapsel des Cryptococcus neo

formans $\quad 305$

Mónus, Z. B., vide K. Kovács.

Moosbrugger, G. A., V. Spuhler und K. Meyer: Über die Ka-

talaseaktivität der Leber beim mit Maul- und Klauen-

seuche infizierten Meerschweinchen. II. Mitteilung über

Katalase 98

- $\quad$ Katalasebestimmungen beim Meerschweinchen. III. Mit

teilung über Katalase 781

Fey, H.: Über Colistämme derO-Gruppe 78 mit einem neuen

B-Antigen bei der Colisepsis des Kalbes .... 543

- Versuch einer Deutung des Prozonenmechanismus mit

Hilfe eines «umgekehrten Blockingtestes». II. Mitteilung 92

Fischer, R., and A. Frew: On Factors Involved in the Me

chanism of Gram-Stain $\quad 88$

Frícsay, M.: Lungeiiveränderungen bei Laboratoriumsratten

infolge Infektion mit Trichosomoides crassicauda . . 351

Friedman, I.: Malignant Nephrosclerosis and Renal Tuberculosis. Report of a case with

granulomatous periarte-riitis nodosa, tuberculous nephritis, and hypertension 13

Gansler, H., et Ch. Rouiller: Modifications physiologiques et

pathologiques du chondriome. Etude au microscope élec-

tronique 217

Gamier, B.: Beitrag zur Morphologie der pathologisch ver-änderten menschlichen Schilddrüse

im Elektronenmi-kroskop. Vergleich mit der normalen und der durch Propylthiouracil

veränderten Rattenschilddrüse . . 129

Glauser, O.: Elektronenmikroskopische Untersuchungen an

Rattenlebern nach Röntgenbestrahlung . . . 150 
Grasset, E., et B. Blondel: Les formes L des Pasteurella:

Facteurs engendrant leurs transformations . . . 598

Hartl, W.: Beobachtungen an pleomorphen Proteuskulturen

unter Einwirkung von Bakteriophagen. (Vorläufige Mit

teilung.) 26

Hermanek, P., und L. Slapak: Über spontane seröse Endo-

karditis des Kaninchens $\quad 318$

Hochali, R.: Der Einfluß einer stabilisierten Amnionflüssig-

keit auf die regenerierende Rattenleber nach partieller

Hepatektomie 288

Kanitz, St., und P. Crovari: Erste Nachrichten über in Li-

gurien gefundene Coxsackie-Viren 620

Kellenberger, G. et E.: Etude de souches colicinogènes au

microscope électronique 582

Knüsel, F., vide W. H. Schopfer.

Koch, A.: Die experimentelle Analyse der Bedeutung der

Symbionten 665

Köhler, H.: Zum sogenannten Mastozytom beim Hund . . 249

Kovac, W., vide H. Moritsch.

Schär, M.a and K. F. Meyer: Studies on Immunization A-gainst Plague. XV. The

Pathophysiologic Action of the Toxin of Pasteurella pestis in Experimental Animals . 51

Schär, M.: Studies on Immunization Against Plague. XVI.

Specific Neutralization of Plague Toxin .... 71

Schleyer, F.: Versuche zur papierelektrophoretischen To-

deszeitbestimmung aus dem Hämolysegrad des Leichen-

serums 273

Schmid, G.: Über den Nachweis von Bang-Agglutininen im

Vaginalsekret von Bang-infizierten Kühen .... 539

Schopfer, W. H., et Th. Posternak: Recherches sur les antiinositols. Action de $\Gamma$ isomytilitol sur Schizosaccharomy-

ces Pombe (Lindner) souche liquefaciens (Osterwalder)

Dekker647

Recherches sur la biochimie des cyclitols. L'utilisa-

tion de trois inososes par six microorganismes . . 654

-, et F. Knüsel: Recherches sur la biosynthèse de la lacto-

flavine. Action du Zinc sur la biosynthèse de cette vita-

mine 659

Shmerlíng, D. H.: Ein Fall von Sarcoma idiopathicum multiplex haemorrhagicum (Morbus

Kaposi) .... 162

Slapak, L., vide P. Hermanek.

Speise Г, P., E. Mezník-Schönbauer und PI. Kürsten: Über die Sekretionsverhältnisse

Neugeborener im Lewis-System. (Blut- und Speicheluntersuchungen an 330 Neugebore-nen nebst Bemerkungen über Befunde an 347 Erwachse-nen und das Lewis-System im allgemeinen.) ...695

Spuhler, V., vide G. A. Moosbrugger. 
SiiГJán, M.: The Large Scale Production of Tetanus Toxin. I. Toxin Production in Media Prepared with Peptic-Tryptic-Ereptic Digested Meat or Casein . . . 311

- The Large Scale Production of Tetanus Toxin. II. Chemical Research in Connection with

Toxin Production . 455

Swierczgńska, Z., vide F. Milgrom.

Tomcsik, J., und /. B. Baumann-Grace: Effekt mechani-

scher Einwirkungen auf die Zellwand gewisser Bazillen 566

Tomellíni, R.: Sur la frequence des anticorps neutralisant

les trois types de virus poliomyélitique en Ligurie . 633

Mooser, H., J. Líndenmann und F. Weyer.· Selektiv-insek-

tizide Wirkung des Butazolidin Geigy

552

Morítsch, H., und W. Kovac: Virologischer und histologi-

sche $\Gamma$ Organbefund eines Wellensittichs bei Psittacosis . 482

Neipp, L., W. Kunz und R. Meier: Versuch zur Analyse der

direkten und indirekten Antiphagenwirkung . . . 331

Neuhold, R.: Phagocvtose lymphatisch leukämischer Zellen

in den Kupfferschen Sternzellen nach Triaethylenmela-

minbehandlung $\quad 748$

Postemak, Th., vide W. H. Schopfer.

Py, G.: L'emploi des flitres en microscopie et microphotographie551

Raska, K., B. Bednár und /. Rotta: Gelenkveränderungen

nach experimentellen Streptokokkeninfektionen bei Ka-

ninchen, welche eine generalisierte Shwartzman-Re-

aktion überlebt haben. II. Mitteilung: Beitrag zu der ex

perimentellen Forschung über Streptokokkeninfektio

nen und ihre Folgen 205

Raska, K., und /. Rotta: Die Persistenz von Streptokokken

der Gruppe A nach intranasaler Infektion .... 356

Rivkine, A.: Action de certains anions tensio-actifs sur le comportement antigénique des

constituants $\mathrm{M}$ et $\mathrm{C}$ des streptocoques hémolytiques du groupe $\mathrm{A}$. . . . Ill

- Influence de certains anions tensio-actifs sur les reac

tions d'agglutination spécifique étudiées sur le système

T des streptocoques hémolytiques du groupe A . . 519 Rotta, J., vide K. Raska. Rouiller, Ch.,

vide H. Gamier. Rüttner, J. R., und H. E. Brunner: Zur Frage einer cancero-

genen Wirkung der Azofarbstoffe Evans Blue und Try-

panblau auf das Retikuloendotheliale System der Leber 436 Rüttner, J. R., H. E. Brunner und A.

P. Vogel: Untersuchun-

gen über die Kupfferschen Zellen der Rattenleber . . 738

Sansonnens, R.: Les suspensions homogènes de Mycobac-

téries dans $\Gamma$ expérimentation in vitro et in vivo . 504

Scevola, M. E., vide A. de BaГbierí.

Schär, B.: Vortrag zum Film «Leukozytotaktische Wir

kung von Bakterienpolysacchariden» 637

- $\quad$ vide P. Desaulles.

Trew, A., vide R. Fischer. 
Vischer, W. A.: Eine Deckglasmethode zur längerdauernden kontinuierlichen Beobachtung von Zell- und Bakterien-

kulturen 560

- $\quad$ Über die Virulenz von Isonicotinsäurehydrazid-resisten-

ten Tuberkelbakterien beim Meerschweinchen . .710

Vogel, A. P., vide J. R. Rüttner. Weyer, F., vide H. Mooser. Zähner, H., vide L. Ettinger. Zogg, H.: Über die Beeinflussung von Pathogenität und

Wachstum eines pflanzlichen Parasite $\pi$ durch die Vit-

amine $\mathrm{Bx}$ und Biotin 501

Zollinge $\Gamma, H$. U.: Stimulation de $\Gamma$ senilen Ratten epidermis

durch stabilisierte Amnion-Salbe 429

- $\quad$ Beschleunigung de $\Gamma$ Wundheilung beim Meerschwein

chen durch amnionhaltige Salbe $\quad 729$

GESELLSCHAFTSBERICHTE - SOCIETY TRANSACTIONS - SOCIÉTËS

Société Suisse de Microbiologie. 15e assemblée annuelle, les 9 et 10 juin

1956, à Lausanne. - Schweizeùsche Mikrobiologische Gesell-

schaft. 15. Jahresversammlung, 9. und 10. Juni 1956, in Lausanne 495

Mitglieder-Liste de $\Gamma$ Schweizerischen Mikrobiologischen Gesellschaft -

Membres de la Société Suisse de Microbiologie .... 687

NECROLOGIA

In memoríam Albert Jan Klugver, 1888-1956 488

BUCHBESPRECHUNGEN -BOOK REVIEWS - LIVRES NOUVEAUX

$125,244,362,491,692,798$

VARIA 248

PERSONALIA 128

Register rerum 802

Register autorum 